\title{
Coronal Magnetic Structure at a Solar Sector Boundary
}

by

John M. Wilcox and Leif Svalgaard

Reproduction in whole or in part

is permitted for any purpose of

tho United States Government.

SUIPR Report No. 547

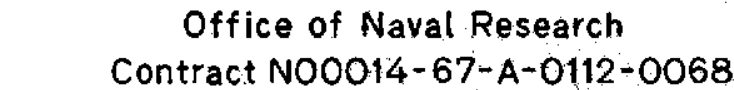

National Aeronautics and Space Administration Grant NGR 05-020-559

$$
\text { and }
$$

National Science Foundation

Grant GA-31138

\section{INSTITUTE FOR PLASMA RESEARCH STANFORD UNIVERSITY, STANFORD, CALIFORNIA}


CORONAL MAGNETIC STRUCTURE AT A SOLAR SECTOR BOUNDARY

by

John M. Wilcox and Leif Svalgaard

\author{
Office of Naval Research \\ Contract N00014-67-A-0112-0068 \\ National Aeronautics and Space Administration \\ Grant NGR 05-020-559 \\ and \\ National Science Foundation \\ Grant GA-31138
}

SUIPR Report No. 547

November 1973

Institute for Plasma Research

Stanford University

Stanford, California

Submitted to Solar Physics

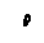

I 
CORONAL MAGNETIC STRUCTURE AT A SOLAR SECTOR BOUNDARY

\author{
John M. Wilcox and Leif Svalgaard \\ Institute for Plasma Research, Stanford University \\ Stanford, California
}

\begin{abstract}
The persistent large-scale coronal magnetic structure associated with a sector boundary appears to consist of a magnetic arcade loop structure extending from one solar polar region to the other in approximately the North-South direction. This structure was inferred from computed coronal magnetic field maps for days on which a stable magnetic sector boundary was near central meridian, based on an interplanetary sector boundary observed to recur during much of 1968 and 1969.
\end{abstract}


CORONAL MAGNETIC STRUCTURE AT A SOLAR SECTOR BOUNDARY

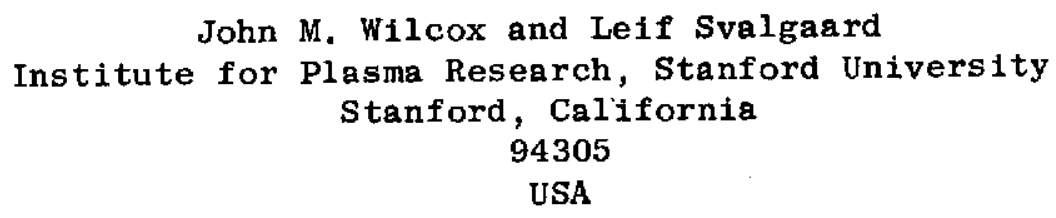

An Atlas comprising 11,000 microfilm maps of the calculated potential (or current-free) magnetic fields of the solar corora between August 1959 and June 1970 has been prepared by Newkirk et al. (1972, 1973). The Atlas was prepared from observations of the line-of-sight component of the photospheric magnetic field obtained by Robert Howard with the 150-foot solar tower at Mount Wilson Observatory. The publishing of this Atlas is a great service to the solar physics community.

The relation of computed coronal magnetic field structures to solar sector boundaries was studied by Newkirk (1972). We have used the Atlas to investigate the coronal magnetic configuration associated with a stable solar and interplanetary magnetic sector boundary (Wilcox, 1968) that recurred regularly during each solar rotation for approximately two years. During 1969 this boundary, as observed by spacecraft magnetometers near the earth, is shown as the dotted line in Figure 1 (Wilcox and Colburn, 1972). The slope of the dotted line corresponds to a rotation period near 28 days. Before the boundary the interplanetary field polarity was away from the sun and after the boundary the polarity was toward the sun.

The average transit time for the solar wind plasma to transport the large-scale solar magnetic field to the earth was observed by Wilcox and Ness (1965) to be approximately $4 \frac{1}{2}$ days. Therefore $4 \frac{1}{2}$ days were subtracted from each time at which this. sector boundary was observed by spacecraft near 
the earth to give a list of times at which the sector boundary should have been near central meridian on the sun. The maps of the computed coronal field corresponding to the times on this list were examined. The average difference between a time on the list and the time of the nearest computed coronal field map was about 0.2 days.

The clearest example of the coronal magnetic field structure associated with this sector boundary is shown in Figure 2. Five sets of maps spaced at intervals of about 1.5 days show the rotation of this sector boundary across the solar disk. The boundary was near central meridian on 1.5 March 1969. For each day two sets of maps are shown. The first set (labeled "general fields") shows the geometry of the coronal magnetic field, and includes field lines originating from both strong and weak fields at the photosphere. The solar photosphere is divided into 648 surface elements of equal area. A field line is plotted from the center of any surface element whenever the calculated field strength at the photosphere exceeds 0.08 Gauss. The second set of maps (labeled "strong fields") shows field lines which emanate from the regions of strongest photospheric magnetic field. Maps are available for every $10^{\circ}$ interval of solar longitude; the maps shown in Figure 2 are at $20^{\circ}$ intervals.

A portion of the weak field map for 1.5 March 1969, when the sector boundary was near central meridian, is re-drawn in Figure 3. This structure composed of a series of magnetic loops has been called a magnetic arcade by Newkirk and Altschuler (1970). The dashed line added to Figure 3 shows the apparent location of the solar magnetic sector boundary. The dashed line is drawn through the middle of the lowest-lying loops. To the west of the boundary the loops are directed out of the sun and to the east of the boundary 
the loops are directed into the sun. This polarity can be clearly seen in the strong field maps of Figure 2, in which field lines out of the sun are labeled with a small 0 at their base and field lines directed into the sun are labeled with a small + sign at their base. Note that this is the reverse of the convention generally adopted for representing the photospheric magnetic field, see for example the Atlas of Solar Magnetic Fields (Howard et al. 1967).

The sector boundary shown with the dashed line in Figure 3 runs from one polar region to the other approximately in the North-South direction. Because of the difficulties of observing in the polar regions of the sun, it is not possible to determine the precise extent in latitude of the boundary, however it appears from Figure 3 that the boundary extends from near one pole to near the other pole.'

The average direction of the boundary shown in Figure 3 is nearly in the North-South direction. The small curvature in the boundary is in the direction opposite to that expected from the effects of differential rotation. The boundary shown in Figure 3 appears to be very similar to the schematic solar sector boundary shown in Figure 4 (Wilcox, 1971), which was obtained from an analysis involving comparison of the observed interplanetary magnetic field with the photospheric magnetic field observed at heliographic latitudes between $40^{\circ}$ North and $40^{\circ}$ South (Wilcox and Howard, 1968): The schematic boundary shown in Figure 4 ends at $40^{\circ}$ latitude only because the regular observations of the photospheric magnetic field on which the analysis was based stopped at about $40^{\circ}$ latitude. In the analysis the boundary was clearly seen at all the latitudes examined, and it was clear that the boundary extended to higher latitudes. 
Coronal magnetic field maps for the days during 1968 and 1969

when this boundary was near central meridian have been examined. The clearest example of the magnetic arcade structure was shown in Figure 2. There is a considerable variation in the clarity with which the structure can be seen during the other days when the boundary was near central meridian. Strong field maps for four other recurrences of the boundary near central mexidian are shown in Figure 5. For the strong field maps the solar photosphere is divided into 2592 surface elements of equal area, and field lines are drawn from the 400 areas having the strongest field. Therefore the fact that the sector magnetic arcades shown in Figure 5 do not cover the complete range of latitudes is probably related to the fact that after the 400 strongest field lines have been drawn, the sector arcade had not been completely defined. The strongest fields associated with the sector arcade appear to be in the northern hemisphere, which has been predominant for most forms of solar activity during the past sunspot cycle.

One of us (LS) scanned through the Atlas of Magnetic Fields in the Solar Corona during the interval from the middle of June 1968 through the end of 1969. The interplanetary boundary was observed to recur in a stable manner through this interval, as can be seen in Figure 1. This scanning was done without knowledge of the positions of the sector boundaries. The central meridian time was recorded for all magnetic arcades that met the following criteria, and were therefore similar to the arcades shown in Figures 2, 3 and 5: 1) Approximately North-South axis, i.e. field lines in the East-West direction, 2) At least one quarter of the disk wide in the East-West direction, 3) At least one-half of the disk long in the 
North-South direction, 4) Visible in both the general and strong field maps, and 5) Can be followed from the East limb through central meridian passage. The polarity of these arcades was recorded according to whether the westward (leading) part of the arcade had field lines directed outward or inward to the sun.

Figure 6 shows a histogram of the number of arcades versus the number of days between central meridian passage of the arcade and the spacecraft observation of the interplanetary sector boundary near the earth. If the interpretation of these coronal magnetic arcades as being related to solar sector boundaries is accepted, then the quantity $\Delta t$ is the transit time for the solar wind plasma to bring the field lines comprising the boundary from the sun to the earth. In Figure 6 the arcades having a westward (leading) polarity out of the sun are cross-hatched. These arcades have the correct magnetic polarity to be associated with the polarity change of the observed interplanetary sector. The two arcades having a westward polarity into the sun are shown without cross-hatching.

The average transit time for the eight arcades at 4 days and 5 days in the histogram having the same polarity as the interplanetary boundary is 3-7/8 days. This is consistent with the transit time of $\left(4 \frac{1}{2} \pm \frac{1}{2}\right)$ days discussed by Wilcox (1968), particularly since the average solar wind velocity would tend to be larger near the maximum of the sunspot cycle (1968 and 1969) than near the minimum, when the $4 \frac{1}{2}$ day transit time was observed. No arcades with polarity opposite to the interplanetary boundary polarity were observed near this transit time, and only two such opposite polarity arcades were observed in the transit time interval from 0 to 10 days, 
as compared with ten arcades in this interval with the same polarity. Thus within the limitations inherent in the small number of cases this analysis supports the identification of the magnetic arcades as described above with the sector boundaries.

If we accept the magnetic arcade structure of Figure 3 as the large-scale coronal structure associated with a sector boundary, several interesting implications can be discussed:

1. The sector boundary extends nearly from pole to pole, i.e., the sector structure has a very large extent in latitude on the sun.

2. The sector boundary is approximately in the North-South direction on the sun. Little or no effects of differential rotation are observed in the shape of the sector boundary. Instead, the sector boundary appears to be in a rigidly rotating coordinate system.

3. The solar sector boundary is not influenced by the solar equator. At any given longitude the sector polarity is the same on both sides of the equator.

4. Let us examine the source of the solar wind plasma as a function of heliographic latitude. The sector arcade structure shown in Figure 3 would appear to have approximately the same conditions for efflux of photospheric plasma outwards at all latitudes. Thus at least as far as the largescale coronal magnetic structure associated with the sector boundary is concerned, there is no evidence for a favored latitude as the source of solar wind plasma, either at equatorial latitudes or at higher latitudes.

5. Let us examine the source of the solar wind plasma as a function of heliographic longitude. The region near the solar sector boundary appears to have closed coronal magnetic field lines which might tend to 
inhibit the outward flow of the solar wind. A few tens of degrees of longitude (or a few days solar rotation) away from the boundary appears to be a region with open coronal field lines through which the solar wind plasma could more readily flow outward. This region with open coronal field lines might have a smaller electron density and be observed as a coronal hole. This interpretation is consistent with that made from a rocket shot by Krieger et a1. (1973) who observed a coronal hole that would have been at central meridian on 22-23 November 1970. A solar sector boundary was at central meridian on 20 November 1970 , since it was observed by spacecraft at the earth on 24 November 1970. Thus the coronal hole observed by Krieger et al. (1973) was two or three days eastward (following) a solar sector boundary. A semblance of the magnetic arcade structure associated with this boundary can be seen in the right hand portion of the coronal magnetic field map for 24 November 1970 shown as Figure 3 of Krieger et a1. (1973).

6. We note that large solar flares are likely to occur near a solar sector boundary (Bumba and Obridko, 1969), 1.e., In a region where the sector magnetic fields are oppositely directed and where conditions for magnetic merging and the release of magnetic energy may be favorable. The direct radial access of cosmic rays accelerated in such flares may be inhibited by the closed sector magnetic arcade structure. Instead, such cosmic rays might flow along the arcade loop structure over a few tens of degrees of longitude until they come near open magnetic field lines near the base of the arcade, and then escape outward into the interplanetary medium. In summary, the persistent large-scale coronal magnetic structure associated with a sector boundary is probably similar to the magnetic arcade structure shown in Figure 3 extending in approximately the North-South 
direction from one polar region to the other. The comparisons of the computed coronal field with eclipse photos suggests to Altschuler and Newkirk (1969) that the calculations on which the structure shown in Figure 3 is based can be expected to give an accurate representation of the shapes and locations of the coronal field lines. To the extent that these two statements are correct, the six implications discussed above would appear to follow. The coronal field maps appear to give a "snapshot" of the rigidly rotating solar magnetic sector structure that was derived earlier from analysis of comparisons of the observed interplanetary and photospheric magnetic fields.

These results are consistent with the preliminary identification by Newkirk (1972) of magnetic arcades with solar sector boundaries.

\section{Acknowledgements}

The solar physics community is greatly indebted to Gordon Newkirk, Jr., Dorothy E. Trotter, Martin D. Altschuler and Robert Howard for making the Atlas of Magnetic Fields in the Solar Corona available. This work was supported in part by the Office of Naval Research under Contract N00014-67A-0112-0068, by the Nationa1 Aeronautics and Space Administration under Grant NGR 05-020-559, and by the Atmospheric Sciences Section of the National Science Foundation under Grant GA-31138. 
References

A1tschuler, M. D. and Newkirk, G., Jr.: 1969, Solar Phys. $\underline{9}, 131$.

Bumba, V. and Obridko, V. N.: 1969, Solar Phys. 6, 104.

Howard, R., Obridko, V. N., and Smith, S. F.: 1967, Atlas of Solar Magnetic

Fields 1959-1966, Carnegie Institution of Washington Publication No. 626.

Krieger, A. S., Timothy, A. F., and Roelof, E. C.: 1973, Solar Phys. 29, 505.

Newkirk, G. Jr., and Altschuler, M. D.: 1970, Solar Phys. 13, 131 .

Newkirk, G. Jr.: 1972, 'Coronal Magnetic Fields and the Solar Wind',

Solar Wind, ed., C. P. Sonett, P. J. Coleman, Jr. and J. M. Wilcox.

National Aeronautics and Space Administration SP-308, Washington, D.C.

Newkirk, G. Jr., Trotter, D. E., Altschuler, M.D. and Howard, R.: 1972,

Solar Phys. 24,370 .

Newkirk, G. Jr., Trotter, D. E., Altschuler, M. D. and Howard, R.: 1973,

A Microfilm Atlas of Magnetic Fields in the Solar Corona, National

Center for Atmospheric Research, Boulder, Colorado, NCAR Technical

Note, NCAR-TN/STR-85.

Wilcox, J. M.: 1968, Space Sci. Rev. $8,258$.

Wilcox, J. M.: 1971, Comments on Astrophys. and Space Phys. $\underline{3}, 133$.

Wilcox, J. M., and Colburn, D. S: 1972, J. Geophys. Res., 77, 751.

Wilcox, J. M., and Howard, R.: 1968, Solar Phys. 5, 564.

Wilcox, J. M. and Ness, N. F.: 1965, J. Geophys. Res., 70, 5793. 


\section{Figure Captions:}

Figure 1. Spacecraft observations during 1969 of the sector boundary discussed in this paper are indicated by the dotted line in this figure, which shows the interplanetary sector structure observed with the Ames Research Center magnetometer on.Explorers 33 and 35. The observed interplanetary field polarity is plotted underneath a chart of planetary magnetic 3-hour range indices $\mathrm{Kp}$ (after Bartels), where dark shading is field polarity toward the sun and light shading is field polarity away from the sun. The hatched shading indicates regions of ambiguous polarity (usually with the field directed approximately at a right angle to the spiral field direction), and blank intervals indicate no available spacecraft observations of the interplanetary field. (Wilcox and Colburn, 1972).

Figure 2 ( $a$ and $b$ ). Coronal magnetic field maps for 1.5 March 1969 when the sector boundary shown in figure 1 was near central meridian, and for intervals of 1.5 days before and after this time. A magnetic arcade loop structure centered about the sector boundary can be seen to rotate with the sun. The dashed line shows a sector boundary drawn through the middle of the lowest loops. The left side of the figure shows "general field" maps in which all field lines coming from photospheric areas with field strength greater than 0.08 gauss are plotted, and the right side of the tigure shows "strong field" maps in which field lines coming from 
the 400 photospheric areas of strongest field are plotted. These maps display different views of the same calculation for the same photospheric data.

Figure 3. The magnetic arcade loop structure from the weak field map of 1.5 March 1969 is redrawn. The dashed line shows a sector boundary drawn through the middle of the lowest magnetic loops. The sector boundary extends from one polar region to the other in approximately the North-South direction.

Figure 4. Schematic of an average solar sector boundary derived from analysis of a comparison of the observed interplanetary magnetic field and the observed photospheric magnetic field. The boundary is approximately in the North-South direction over a wide range of latitude. The solar region to the west of the boundary tends to be quiet and the region to the east of the boundary tends to be active.

Figure 5. Coronal magnetic field maps for four days in which the sector boundary was near central meridian. This figure shows the persistence of the sector coronal magnetic arcade structure during $1 \frac{1}{2}$ years. These "strong field" maps show only field lines emerging from 400 photospheric areas having the strongest fields, so that the full extent in latitude of the magnetic arcades are not shown.

Figure 6. Histogram of the number of arcades satisfying criteria described in the text versus the number of days $\Delta t$ between central meridian passage of the arcade and the spacecraft observation of the interplanetary sector boundary near the earth. If the arcades 


\begin{abstract}
are identified with solar sector boundaries then $\Delta t$ is the sun-earth transit time of the boundaries. The interval $0<\Delta t \leq 1$ day is labeled 1 day, et cetera.
\end{abstract}




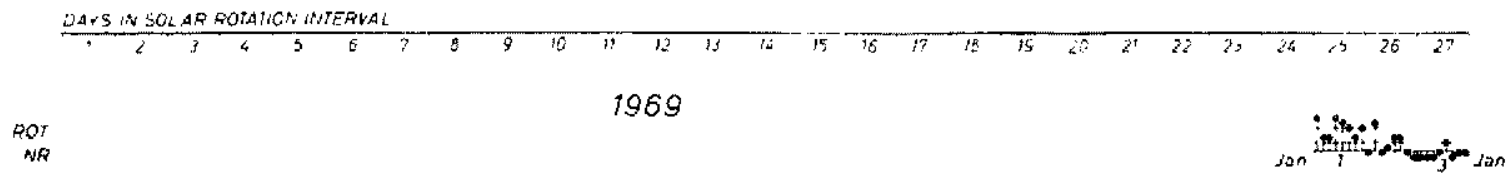

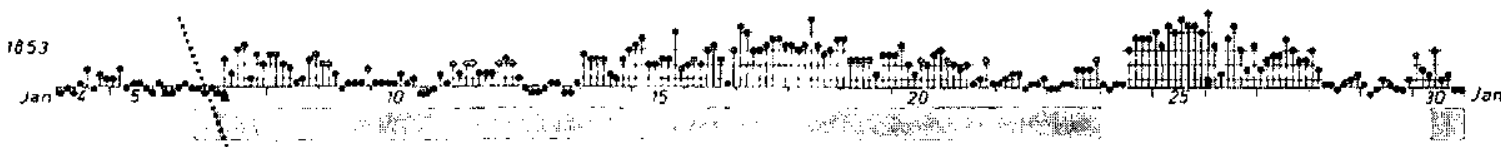

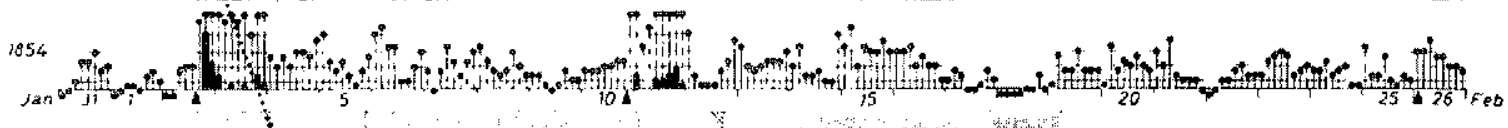
Fes

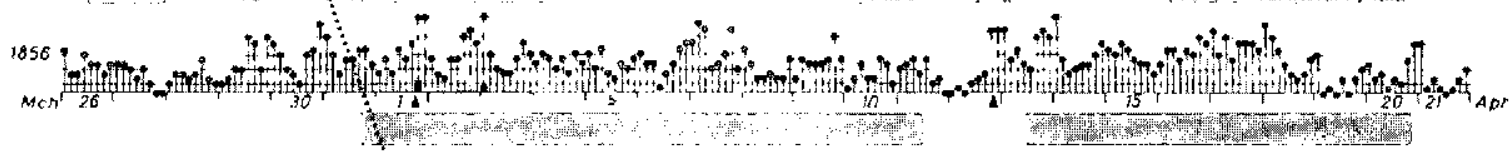

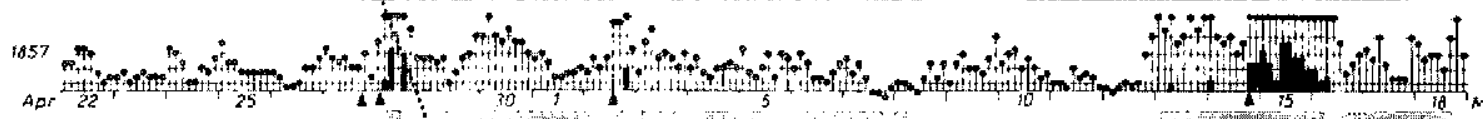

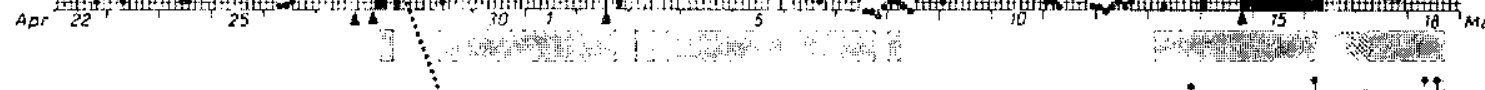
Mays

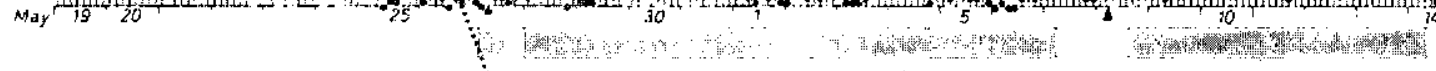

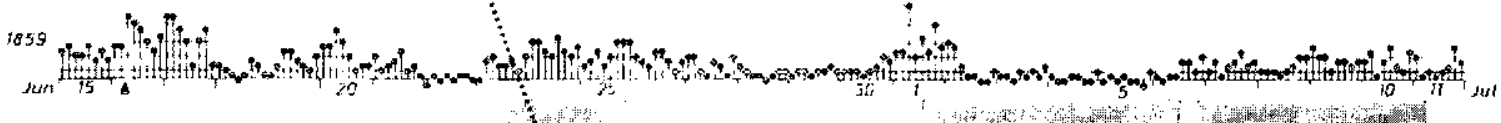
${ }_{3 u}^{1000} \mathrm{H}_{12}$

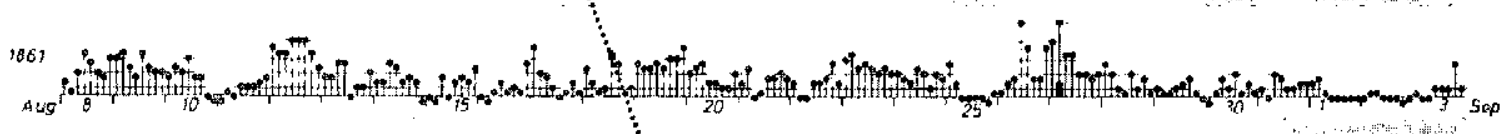

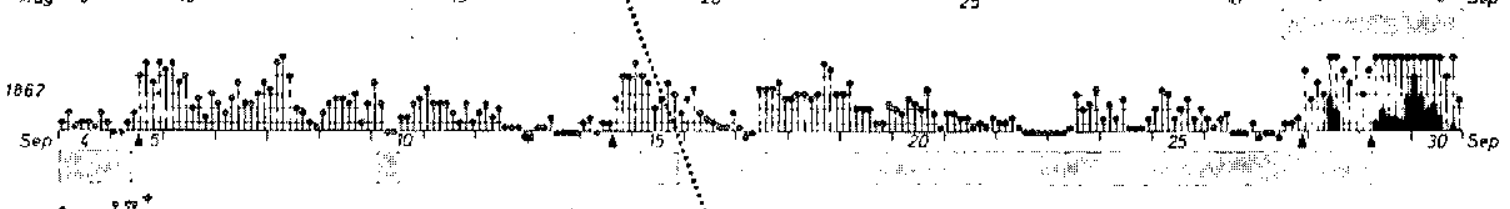

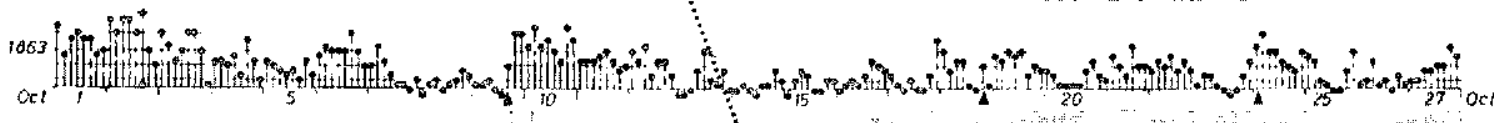

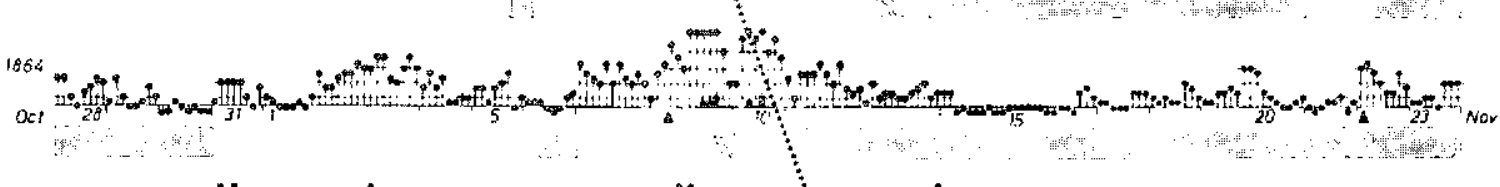

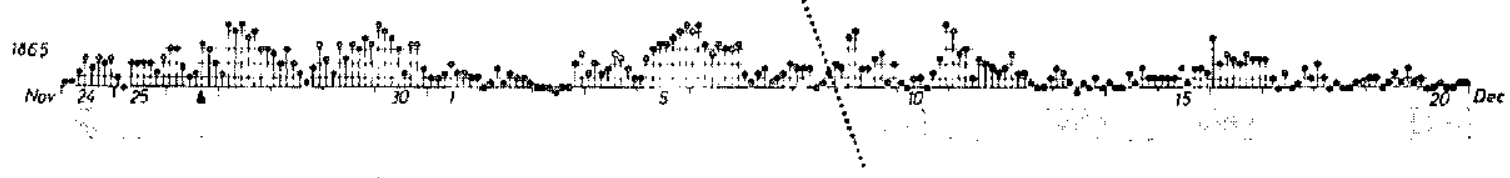
Dec $2 T^{2}$

PLANETARY MAGNETIC THREE - HOUR - RANGE INOICES KP 1969 Figure $1 \quad, \quad \begin{gathered}k E Y \\ \ldots+\cdots\end{gathered}$ 
COMPUTED CORONAL MAGNETIC FIELD
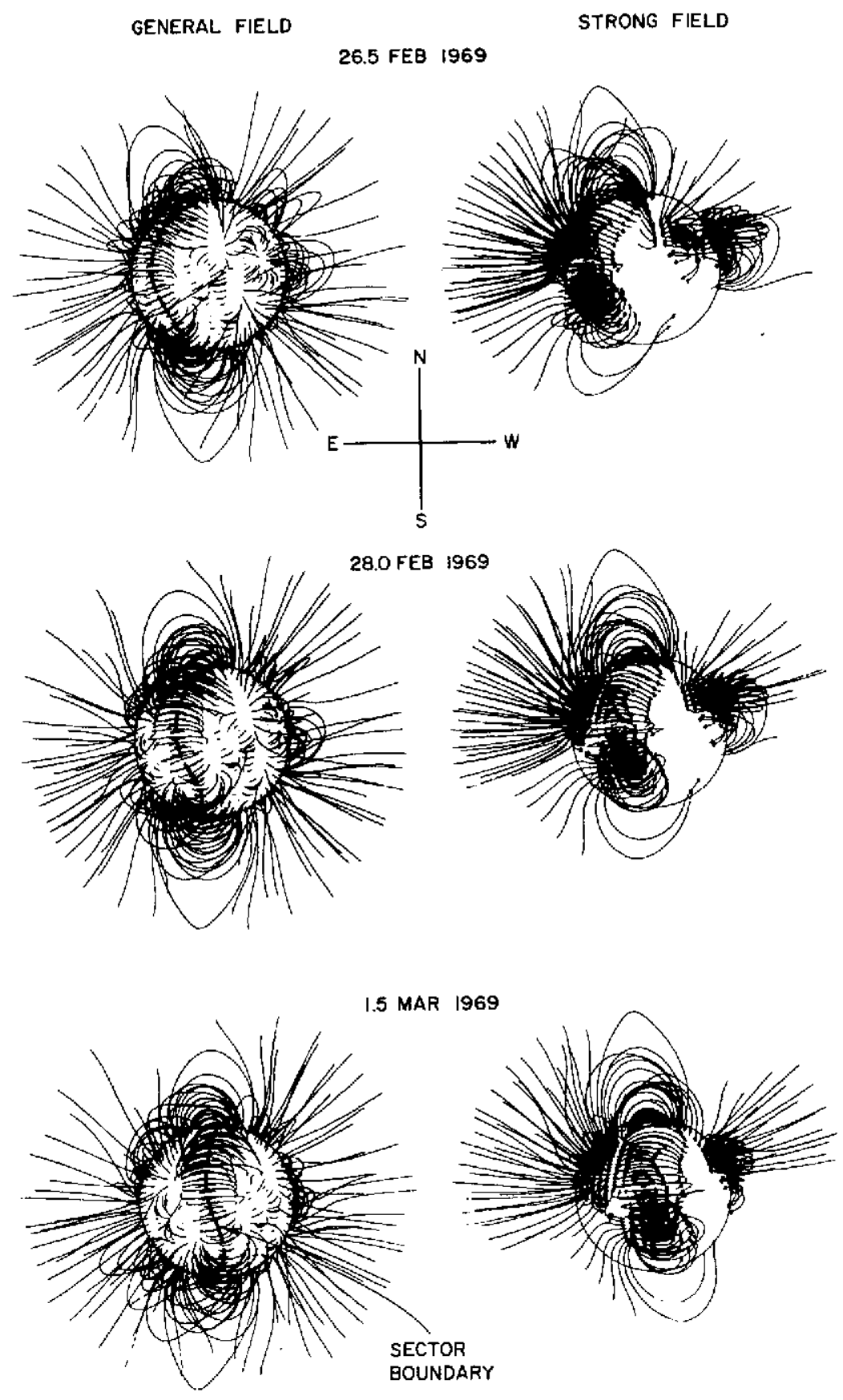

Figure 2 a 


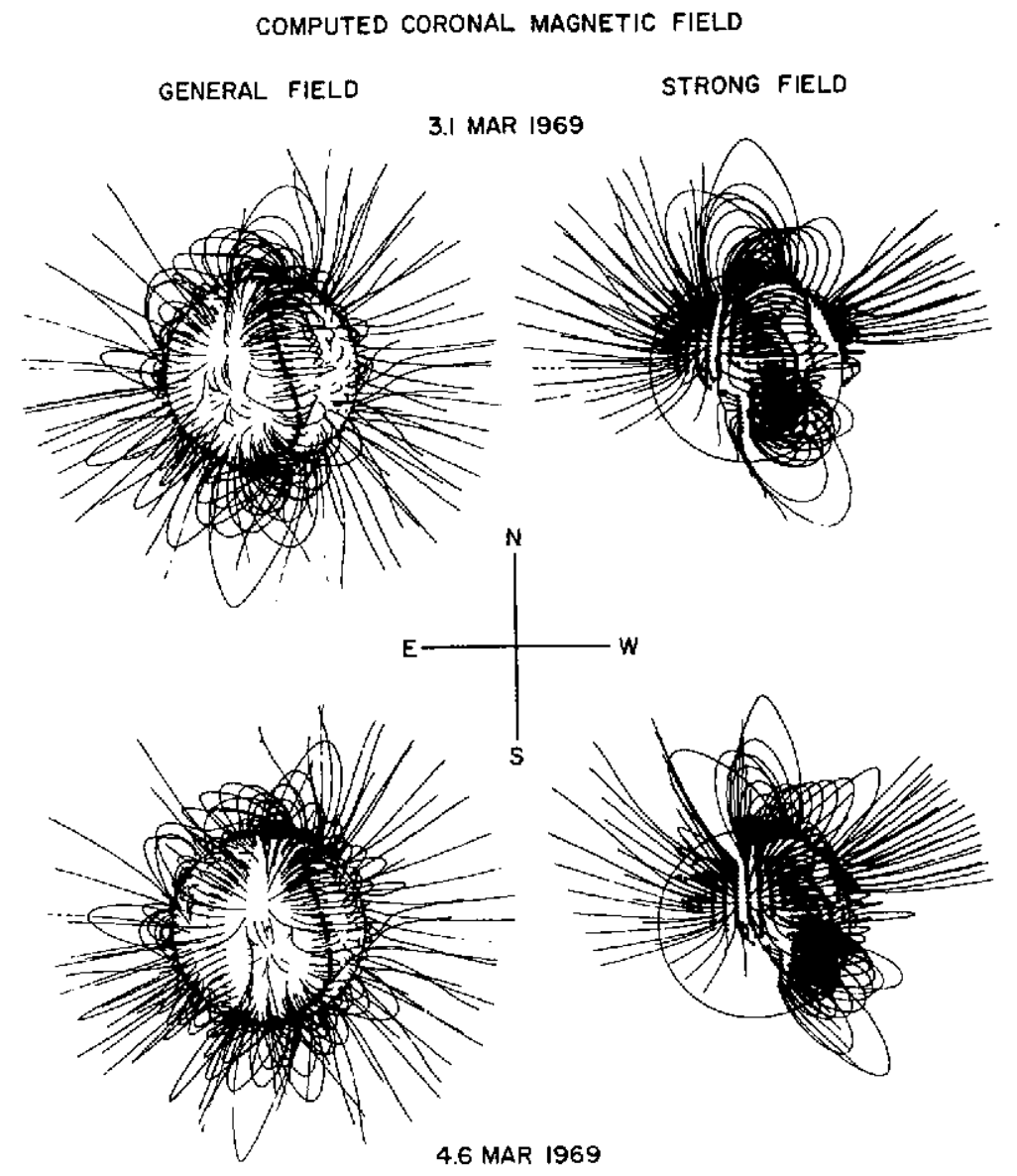

F1gure 2b 


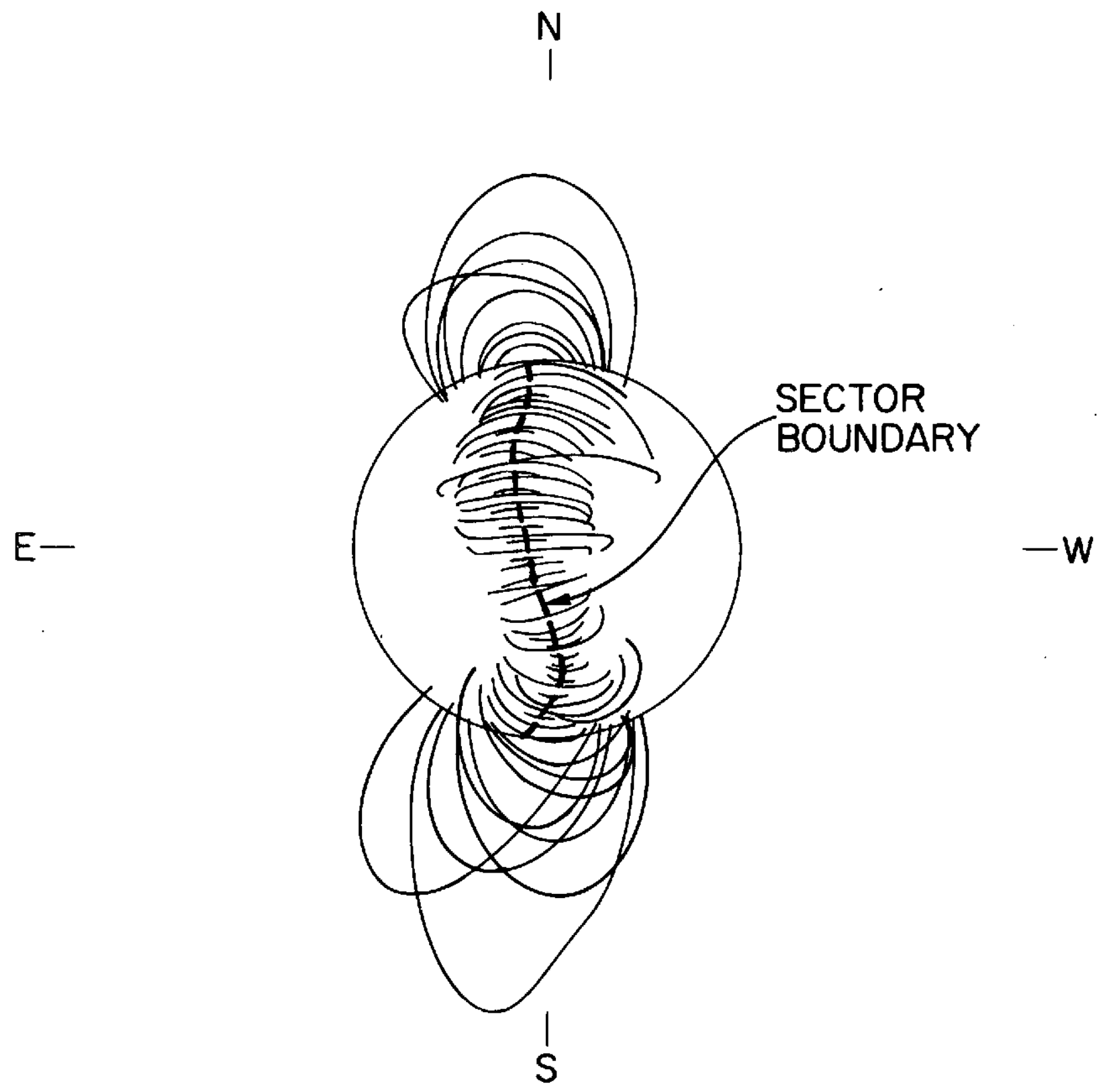

Figure 3 


\section{SCHEMATIC OF SOLAR SECTOR BOUNDARY}
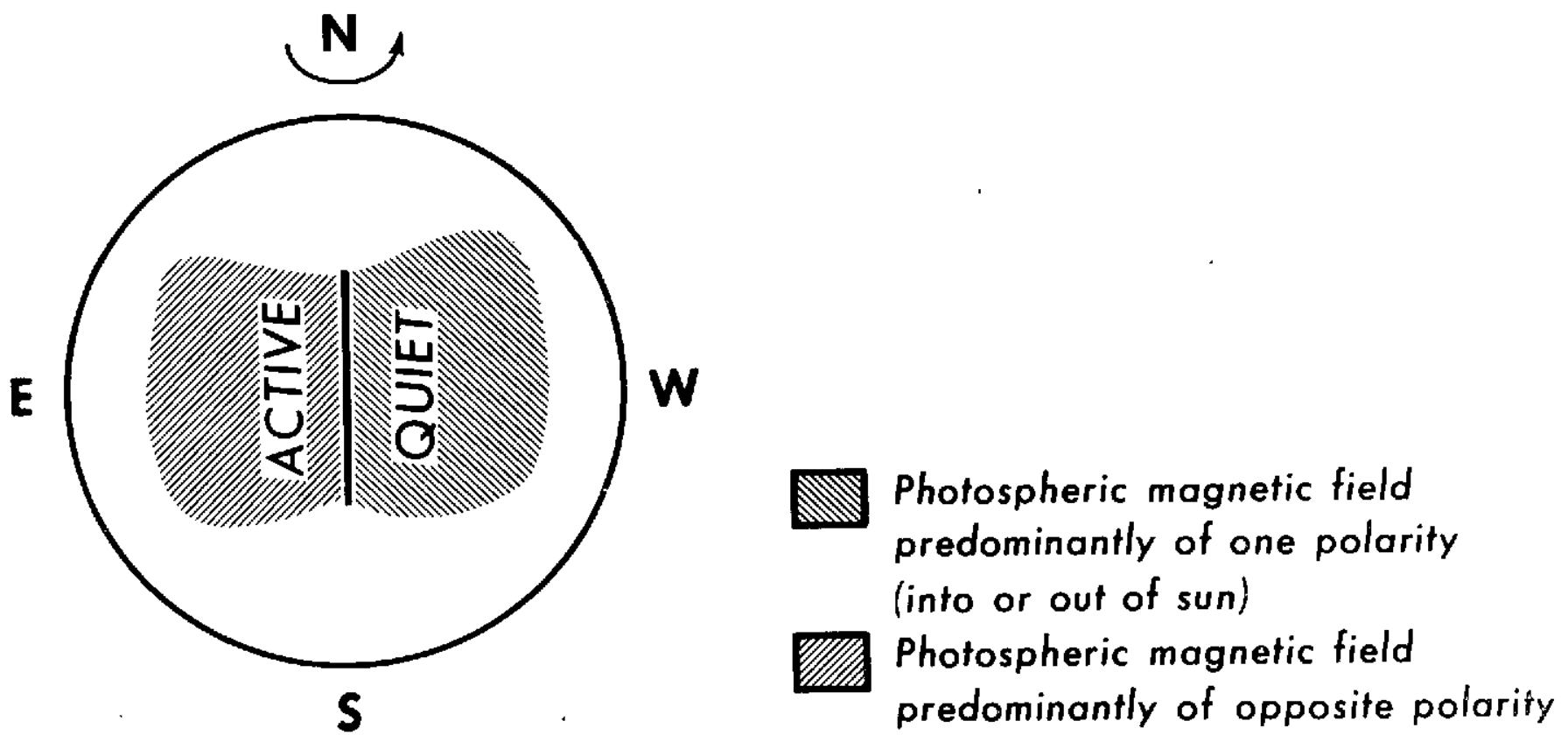

Figure 4 
COMPUTED CORONAL MAGNETIC FIELD

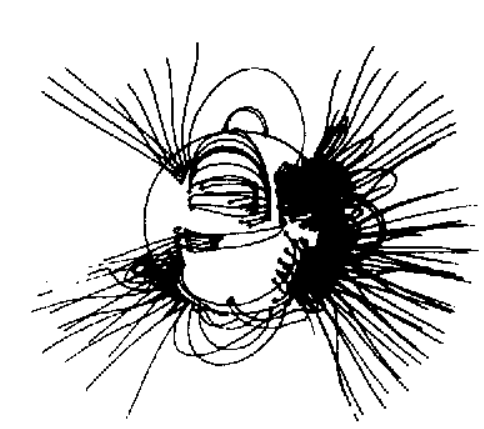

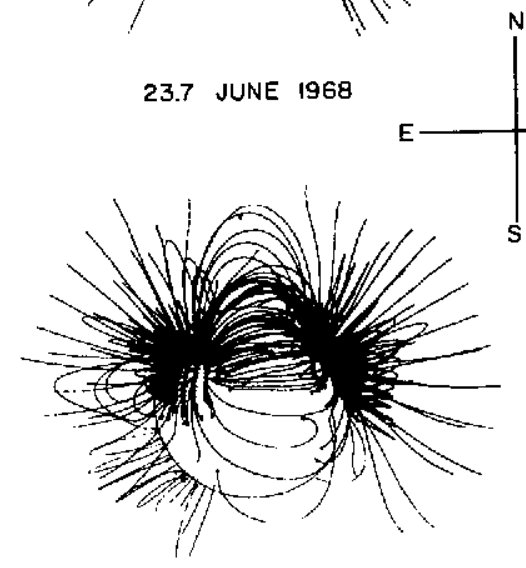

25.1 APRIL 1969

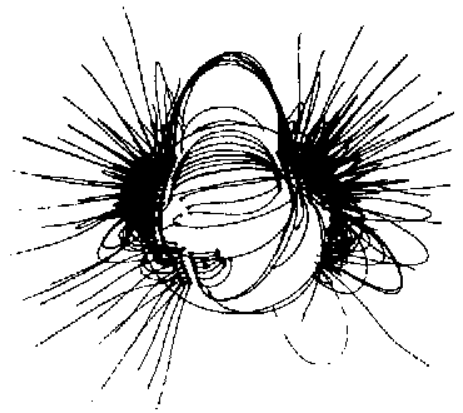

27.3 MARCH 1969

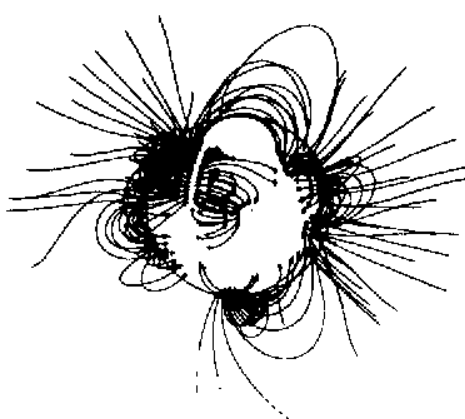

4.4 DEC 1969

Figure 5 


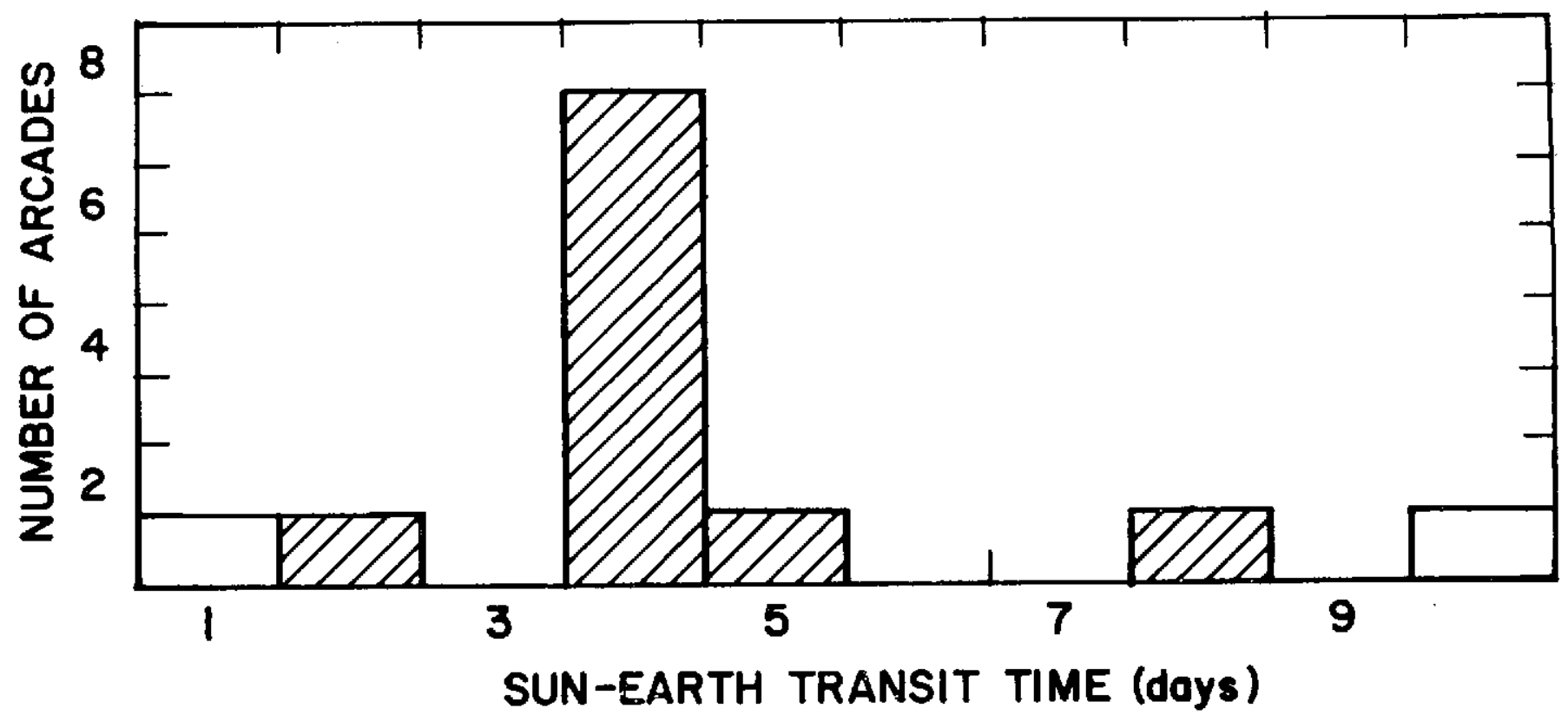

Figure 6 
UNCLASSIFIED

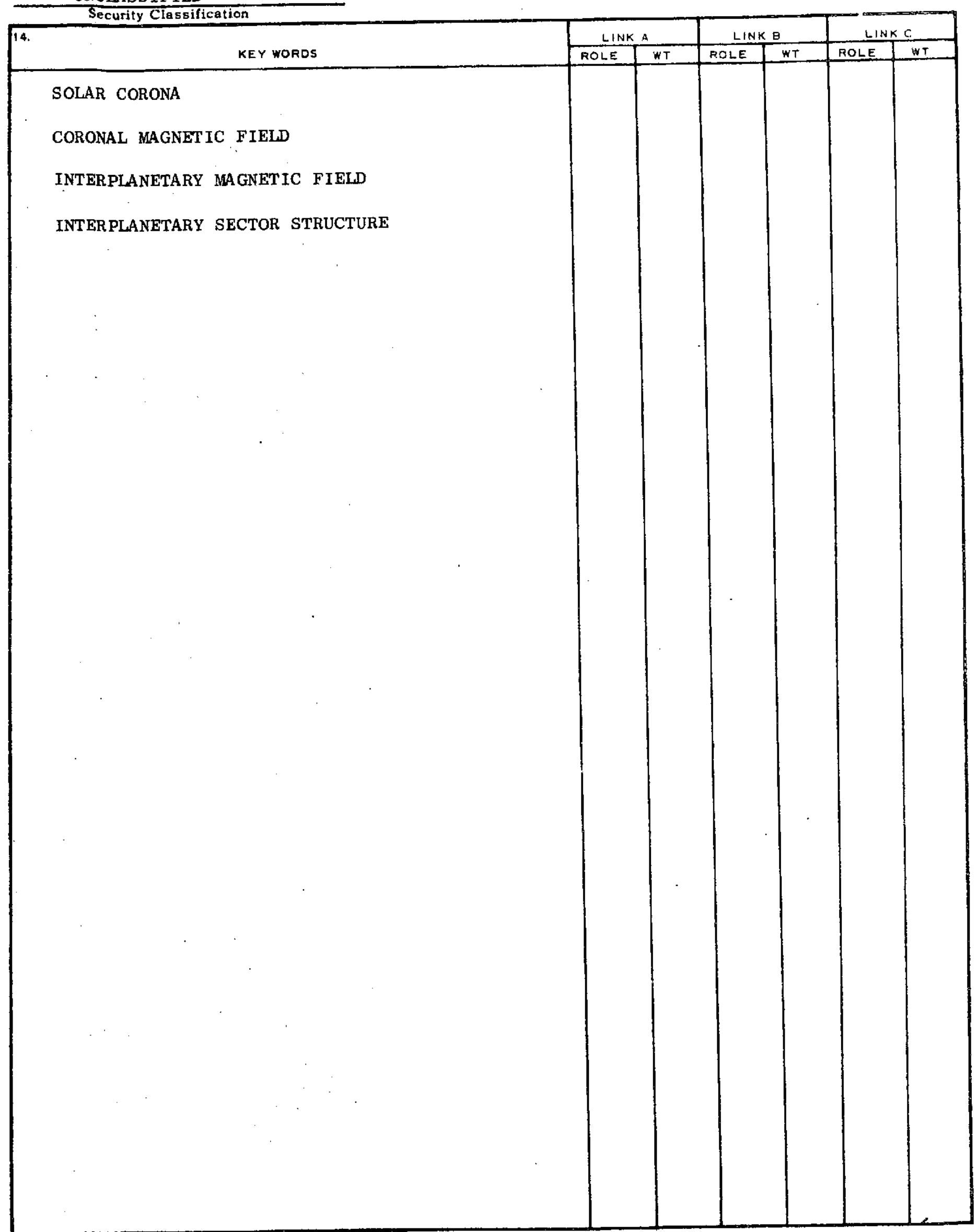


UNCLASS IFIED

Security Classification

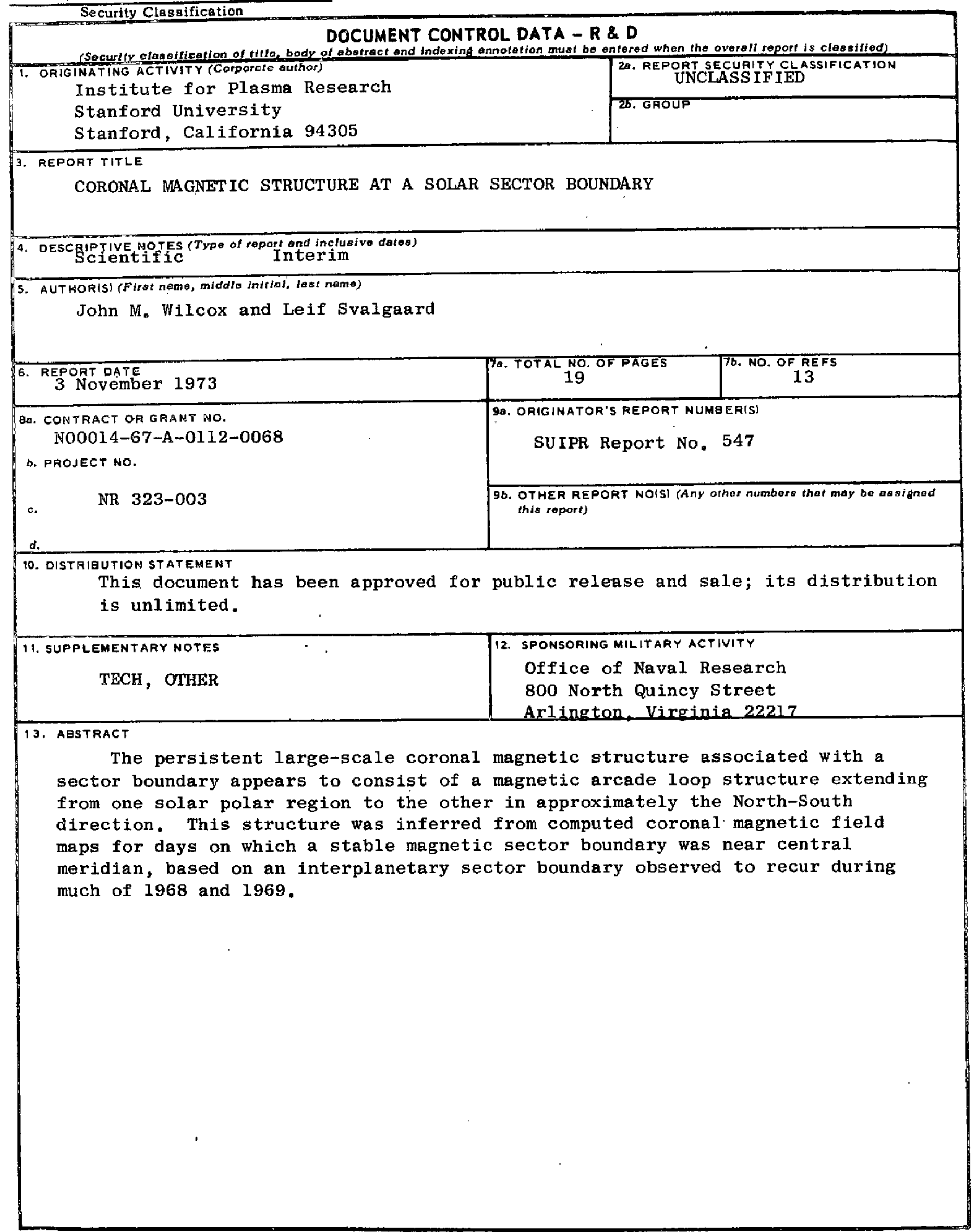

